\title{
Biofilm Formation of Candida albicans Facilitates Fungal Infiltration and Persister Cell Formation in Vaginal Candidiasis
}

\begin{abstract}
Xueqing Wu ${ }^{1,2 t}$, Sisi Zhang ${ }^{1+}$, Haiying $\mathrm{Li}^{2}$, Laien Shen ${ }^{1}$, Chenle Dong ${ }^{1}$, Yao Sun ${ }^{3}$, Huale Chen ${ }^{3}$, Boyun $\mathrm{Xu}^{1}$, Wenyi Zhuang' ${ }^{2}$, Margaret Deighton ${ }^{4}$ and Yue Qu ${ }^{3,5 *}$

'Department of Obstetrics and Gynecology, The First Affiliated Hospital of Wenzhou Medical University, Wenzhou, China, ${ }^{2}$ Department of Obstetrics and Gynecology, Shenzhen University General Hospital, Shenzhen, China, ${ }^{3}$ Department of Laboratory Medicine, The First Affiliated Hospital of Wenzhou Medical University, Wenzhou, China, ${ }^{4}$ School of Applied Sciences, RMIT University, Bundoora, VIC, Australia, ${ }^{5}$ Wenzhou Medical University-Monash BDI Alliance in Clinical and Experimental Biomedicine, Monash University, Clayton, VIC, Australia
\end{abstract}

Background: Vaginal candidiasis is an important medical condition awaiting more effective treatment. How Candida albicans causes this disease and survives antifungal treatment is not yet fully understood. This study aimed to establish a comprehensive understanding of biofilm-related defensive strategies that $C$. albicans uses to establish vaginal candidiasis and to survive antifungal treatment

Methods: A mouse model of vaginal candidiasis was adopted to examine the formation of biotic biofilms on the vaginal epithelium and fungal infiltration by laboratory and clinical strains of $C$. albicans. Histopathological changes and local inflammation in the vaginal epithelium caused by $C$. albicans of different biofilm phenotypes were compared. Antifungal susceptibility testing was carried out for C. albicans grown as planktonic cells, microplate-based abiotic biofilms, and epithelium-based biotic biofilms. Formation of persister cells by $C$. albicans in different growth modes was also quantified and compared.

Results: C. albicans wild-type reference strains and clinical isolates, but not the biofilm-defective mutants, formed a significant number of biotic biofilms on the vaginal epithelium of mice and infiltrated the epithelium. Biofilm formation and epithelial invasion induced local inflammatory responses and histopathological changes in the vaginal epithelium including neutrophil infiltration and subcorneal microabscesses. Biofilm growth on the vaginal epithelium also led to high resistance to antifungal treatments and promoted the formation of antifungal-tolerant persister cells.

Conclusion: This study comprehensively assessed biofilm-related microbial strategies that $C$. albicans uses in vaginal candidiasis and provided experimental evidence to support the important role of biofilm formation in the histopathogenesis of vaginal candidiasis and the recalcitrance of the infection to antifungal treatment.

Keywords: vaginal candidiasis, recurrent vaginal candidiasis, biofilm formation, fungal infiltration, persister cells, antifungal tolerance, mouse model 


\section{INTRODUCTION}

Vaginal candidiasis is one of the commonest medical conditions affecting otherwise healthy women of reproductive age. It was estimated that $23-49 \%$ of women of reproductive age suffer from vaginal candidiasis, with the majority having uncomplicated infections (less than 3-4 episodes within a 12-month period) and a considerable number (6-9\% of the patients) presenting with recurrent infections (at least 3-4 episodes in 1 year); the latter is a stubborn condition characterized by complex pathogenicity and tolerance to antifungal treatment (Ilkit and Guzel, 2011; Sobel, 2016; Blostein et al., 2017). Although vaginal candidiasis is believed to be an immunopathological condition of the human vagina with "neutrophy anergy" as the underlying mechanism, invading microorganisms might still play an important role in its pathogenicity (Yano et al., 2018). Candida albicans is the leading agent causing both uncomplicated and recurrent vaginal candidiasis (Liu et al., 2014; Goncalves et al., 2016). Proposed virulence determinants of $C$. albicans involved in the pathogenesis of vaginal candidiasis include fungal morphogenesis, adhesion to vaginal epithelial cells, the production of phospholipases and proteinase such as secreted aspartyl proteases (Saps), and the presence of candidalysin, a well-identified secreted cytolytic peptide toxin encoded by ece1 (Kalkanci et al., 2013; Richardson et al., 2017).

While much has been done to define host- and pathogenrelated factors contributing to the establishment of vaginal candidiasis (Ilkit and Guzel, 2011; Bruno et al., 2015; Yano et al., 2018), little effort has been placed on microbial mechanisms driving vaginal candidiasis to resist antifungal treatment and further develop into a more troublesome recurrent infection. Despite the fact that most $C$. albicans isolates from patients with uncomplicated or recurrent vaginal candidiasis showed sensitivities to many first-line antifungals, clinical ineffectiveness of these drugs in eradicating Candida or curing recurrent vaginal candidiasis has been constantly reported (Richter et al., 2005; Gamarra et al., 2014; Nagashima et al., 2016; Ying et al., 2016; Adjapong et al., 2017). This suggests that in addition to the host-related factors such as different host sensitivity to pathological responses, microbial strategies other than intrinsic resistance might be involved in the pathogenesis and persistence of vaginal candidiasis (Peters et al., 2014; Sobel, 2016).

Candida albicans and its related species are known to be able to adopt a major pathogenicity strategy, biofilm formation, to initiate and maintain infections, in particular on patients with breached immune defense (Hagerty et al., 2003; Kojic and Darouiche, 2004). Indeed, the role of epithelium-associated C. albicans biofilm in the development of vaginal candidiasis has been proposed (Harriott et al., 2010; Rodriguez-Cerdeira et al., 2019), though a recent study by Swidsinski et al. (2019) did not detect evident biofilms from the vaginal biopsies from patients with confirmed vaginal candidiasis. Two different types of biofilms might be involved in vaginal candidiasis: abiotic biofilms that require a plastic or metal substratum, such as intrauterine devices (IUDs; Auler et al., 2010;
Cakiroglu et al., 2015), and biotic biofilms that use the vaginal epithelium as the supporting base (Harriott et al., 2010; Muzny and Schwebke, 2015). Epithelium-associated biotic biofilms were considered to be more important in the pathogenesis of recurrent vaginal candidiasis, as many patients suffer from recurrent infections without any IUDs implanted or after the removal of IUDs (Cakiroglu et al., 2015). Using a mouse vaginal candidiasis model, Harriott et al. (2010) successfully established C. albicans biofilms on the vaginal epithelium and raised the hypothesis that formation of epithelium-associated biofilms may be an initiating event for the infection (Harriott et al., 2010). A direct link between the formation of epithelium-associated biofilms and histopathological change of the vaginal epithelium, however, is still missing. Although antifungal resistance of Candida biofilms formed by clinical isolates from patients with vaginal candidiasis has been reported, the tests were mostly done on abiotic biofilms prepared in 96-well microplates (Gao et al., 2016; Sherry et al., 2017). Epithelium-associated biotic biofilms grown in a dynamic vaginal environment might differ from in vitro abiotic biofilms in many different ways, including their susceptibility to conventional antifungals (Bjarnsholt et al., 2013).

The typical dynamics of recurrent vaginal candidiasis coincides with the relapsing biofilm infection model proposed by Lewis (2010), suggesting possible involvement of persister cells (Lewis, 2010). Many studies, including our own, have found that persister cells are responsible for antimicrobial tolerance of many biofilm-related bacterial infections (Qu et al., 2010; Yang et al., 2015). Lafleur et al. (2006) first isolated Candida persister cells and linked their formation to the adherence growth mode of C. albicans. It is thus reasonable to hypothesize that biotic biofilms grown on the vaginal epithelium also harbor a small number of persister cells. Treatment of persister cells is known to be troublesome. Conventional antimicrobial agents, unless used at very high dose for an extended period, will not eradicate persister cells residing in biofilms (Yang et al., 2015; Wu et al., 2019). Our study aimed to establish a comprehensive understanding of the importance of C. albicans biofilm formation in the pathogenesis of vaginal candidiasis, in order to identify specific microbial targets for more efficient antifungal therapies.

\section{MATERIALS AND METHODS}

\section{Ethics Statement}

This study was approved by the Ethics Committee of Wenzhou Medical University, China (Ethics approval number: Wydw20160214). All experiments were performed in accordance with the National Institutes of Health guide for the care and use of laboratory animals.

\section{Strains and Identification}

Two biofilm-producing C. albicans laboratory reference strains, C. albicans DAY185 and C. albicans DAY286, and their biofilmdefective mutant strains, $\operatorname{med} 31 \Delta \Delta$ and $\operatorname{bcr} 1 \Delta \Delta$, were used in this study (Lafleur et al., 2006; Uwamahoro et al., 2012). 
Two C. albicans clinical isolates designated as VVC2 and VVC4 were also included. These two isolates were from patients with clinically diagnosed vaginal candidiasis (two and four episodes within a 12-month period, respectively) at The First Affiliated Hospital of Wenzhou Medical University. The clinical isolates were identified to a species level using the following tests: CHROMagar Candida medium (CHROMagar, Paris, France) and the Vitek matrix-assisted laser desorption/ionization-time of flight mass spectrometry (MALDI-TOF MS, bioMérieux, Craponne, France).

\section{Mouse Vaginal Epithelial Biofilm Assay}

An in vivo model of vaginal epithelium-associated biofilms was used essentially as previously described (Harriott et al., 2010). Firstly, BABL/C female mice of $6-8$ weeks old were administered $0.1 \mathrm{mg}$ of estrogen (17 $\beta$-estradiol; Sigma) dissolved in $0.1 \mathrm{ml}$ of sesame oil subcutaneously for three consecutive days prior to inoculation with C. albicans. C. albicans cells were grown in yeast peptone dextrose broth (YPD, $200 \mathrm{rpm}, 30^{\circ} \mathrm{C}$ ) for $20 \mathrm{~h}$. One hundred microliters of fungal suspensions containing $7 \times 10^{5}$ colony-forming units (CFU) of C. albicans cells was used to infect mice via an intravaginal pathway. Mice were maintained in an animal facility and euthanized after $72 \mathrm{~h}$ before the vagina was removed. Fungal growth on the vaginal epithelium and underlying tissue was assessed qualitatively using scanning electronic microscopy (SEM, see below) and quantitatively with the CFU-based viable count method.

\section{Scanning Electronic Microscopy}

For SEM, dissected mouse vaginal tissues were fixed with glutaraldehyde for $2 \mathrm{~h}(2.5 \%, \mathrm{v} / \mathrm{v}$, in $0.1 \mathrm{M}$ cacodylate buffer, $\mathrm{pH} 7.0)$ at room temperature, and dehydrated with gradually increased ethanol levels (50, 75, 85, 95, 100, and absolute 100\%) (Gargani et al., 1989). Samples were coated with gold using a Balzers SCD005 sputter coater and viewed under a scanning electron microscope (SEM; Hitachi, H-7500, Japan).

\section{CFU-Based Viable Counts}

For CFU-based viable counts, dissected mouse vaginal tissues were weighted, homogenized in PBS using a tissue homogenizer, followed by vortexing vigorously for $30 \mathrm{~s}$ four times and sonication at $42 \mathrm{kHz}$ for $10 \mathrm{~min}$. The suspensions were serially diluted with PBS and plated on YPD for $72 \mathrm{~h}$ for enumeration of CFUs.

\section{Histopathology}

For histopathological study of the infected vagina, tissues were fixed in $4 \%$ paraformaldehyde and selected tissue blocks were processed using a routine overnight cycle in a tissue processor. The tissue blocks were then embedded in wax, serially sliced into $5-\mu \mathrm{m}$ sections. The transverse sections were stained with Periodic acid-Schiff (PAS) staining for the presence of C. albicans yeast/hyphae cells or Hematoxylin-Eosin ( $\mathrm{H} \& \mathrm{E}$ ) staining for tissue damage, and then imaged under a light microscope (Nikon ECLPSE 80i, Tokyo, Japan).

\section{Enzyme-Linked Immunosorbent Assay}

Concentrations of two representative inflammatory effectors in mouse vaginal lavage fluids, innate cytokine IL- $1 \beta$ and an alarmin S100A8, were analyzed using commercially available enzymelinked immunosorbent assay (ELISA) kits (Boyun Biotech Co., Ltd, Shanghai, China) per the instructions from the manufacturer (Peters et al., 2014). Enzyme immunoassay/radioimmunoassay (EIA/RIA) plates were incubated with dilutions of lavage fluid (at 1:10 for IL-1 $\beta$ and 1:100 for S100A8) and serially diluted protein standards for $2 \mathrm{~h}$. After washing, the plates were treated with biotinylated polyclonal goat anti-mouse IL$1 \beta$ or S100A8 antibodies for $2 \mathrm{~h}$, followed by incubation with streptavidin horseradish peroxidase (HRP) for $20 \mathrm{~min}$. A tetramethylbenzidine- $\mathrm{H}_{2} \mathrm{O}_{2}$ substrate solution was added to the plates and the reactions were measured with a microplate reader at $450 \mathrm{~nm}$.

\section{In vitro Biofilm Formation on IUDs}

Candida albicans biofilms were grown on two copper IUDs for qualitative analysis. Intrauterine devices were pretreated with fetal bovine serum (Sigma, North Ryde, Australia) overnight at $37^{\circ} \mathrm{C}$ with gentle shaking (75 rpm), washed twice with PBS, and transferred to a 24 -well plate containing $1 \mathrm{ml}$ of freshly prepared fungal suspensions ( $3 \times 10^{7}$ cells/ml in RPMI-1640 at $\left.\mathrm{pH} 4.0\right)$. The plate was incubated for $1.5 \mathrm{~h}$ at $37^{\circ} \mathrm{C}$ with gentle shaking (75 rpm) to allow the yeast cells to adhere to the surfaces. The IDUs were then gently washed with PBS and transferred to a new 24-well plate with RPMI-1640 media ( $\mathrm{pH} 4.0$ ), followed by incubation at $37^{\circ} \mathrm{C}$ with shaking $(75 \mathrm{rpm})$ for $48 \mathrm{~h}$. The established biofilms were examined with SEM. Two IUDs were used in this study; an indomethacin releasing round copper IUD (Medsuture, Shanghai, China), and a yuan-gong type copper IUD (Yantai Jishengyaoxie Co., Ltd., Shangdong, China).

\section{Antifungal Susceptibility Tests for Planktonic Cells and Abiotic Biofilms}

Minimum inhibitory concentrations (MICs) were determined using the broth microdilution method according to CLSI guidelines M27-A3. Drug concentrations ranged from 0.125 to $64 \mu \mathrm{g} / \mathrm{ml}$ for clotrimazole and nystatin and $0.025-128 \mu \mathrm{g} / \mathrm{ml}$ for amphotericin B. The MIC was defined as the concentration resulting in complete growth inhibition for amphotericin B and an inhibition of at least $50 \%$ of fungal growth for other drugs, corresponding to a score of 2 in the NCCLS M27-A3 protocol. Abiotic biofilm MICs were determined by challenging C. albicans biofilms pre-grown in 96-well microplates with antifungal agents prepared in RPMI-1640 for $24 \mathrm{~h}$ (Qu et al., 2016). 3-Bis-(2-methoxy-4-nitro-5-sulfophenyl)-2Htetrazolium-5-carboxanilide (XXT, Sigma-Aldrich, Australia) was used to assess the inhibitory efficacy of antifungal agents on the established abiotic biofilms. Biofilm $\mathrm{MIC}_{80}$ was determined as the lowest concentration that inhibited $\geq 80 \%$ of fungal biofilm growth. RPMI-1640 of $\mathrm{pH} 7.2$ and $\mathrm{pH}$ 4.0 were used as growth medium, respectively, to examine the effect of different environmental acidity on antifungal efficacy as local $\mathrm{pH}$ has been found to impact on the 
antifungal-mediated killing of C. albicans (Ilkit and Guzel, 2011; Kasper et al., 2015).

\section{Ex vivo Antifungal Susceptibility Tests for Biotic Biofilms}

For ex vivo antifungal susceptibility testing, epitheliumassociated C. albicans biofilms were grown for $72 \mathrm{~h}$ before removal from the euthanized animals. Concentrations of selected antifungals ranged from 2 to $32 \mu \mathrm{g} / \mathrm{ml}$ for nystatin, 0.5$1280 \mu \mathrm{g} / \mathrm{ml}$ for clotrimazole, and $1-16 \mu \mathrm{g} / \mathrm{ml}$ for amphotericin B. Infected vaginal tissues were sectioned into small blocks of $3 \mathrm{~mm} \times 5 \mathrm{~mm} \times 5 \mathrm{~mm}$ and exposed to antifungal agents prepared at selected concentrations in RPMI-1640 (pH 4.0 and $\mathrm{pH}$ 7.2, respectively) for $24 \mathrm{~h}$. The treated vaginal tissue blocks were washed with PBS, homogenized with tissue homogenizer. Colony-forming units counting was carried out by plating an aliquot onto YPD plates followed by an incubation at $37^{\circ} \mathrm{C}$ for $48 \mathrm{~h}$. Vaginal tissue treated with RPMI-1640 served as untreated control.

\section{Quantification of Persister Cells From Planktonic Cultures, Abiotic Biofilms, and Biotic Biofilms}

To isolate persister cells from planktonic cultures, suspensions of single cells from cultures at mid-log phase were adjusted to densities of $\sim 3 \times 10^{7} \mathrm{CFU} / \mathrm{ml}$ and exposed to amphotericin B at $100 \mathrm{mg} / \mathrm{ml}$ for $24 \mathrm{~h}$ (Lafleur et al., 2006). To isolate the persister cell population from abiotic biofilms, in vitro biofilms of $C$. albicans were grown in 6-well microplates and collected using a cell scraper. Collected biofilms were then re-suspended into suspensions of single cells at densities of $\sim 3 \times 10^{7} \mathrm{CFU} / \mathrm{ml}$ (LaFleur et al., 2010). Biofilm cells were statically exposed to amphotericin B at $100 \mathrm{mg} / \mathrm{ml}$ for $24 \mathrm{~h}$. To isolate the persister cell population in epithelium-associated biotic biofilms, vagina tissues were removed from the euthanized animals and then sectioned into small blocks of $3 \mathrm{~mm} \times 5 \mathrm{~mm} \times 5 \mathrm{~mm}$ and homogenized with tissue homogenizer. Obtained suspensions were adjusted to densities of $\sim 3 \times 10^{7} \mathrm{CFU} / \mathrm{ml}$ and statically challenged with amphotericin B at $100 \mathrm{mg} / \mathrm{ml}$ for $24 \mathrm{~h}$. To avoid antifungal carryover and accurately quantify the number of persister cells, the antifungal treated suspensions were centrifuged at $3000 \mathrm{~g}$ for $5 \mathrm{~min}$, washed twice with PBS, and resuspended to the same volume with YPD broth. Colony-forming units counting was done by plating serially diluted aliquots onto YPD plates followed by incubation at $35^{\circ} \mathrm{C}$ for $72 \mathrm{~h}$ to maximize recovery of persister cells. The percentage of persister cells in different populations was calculated as follows: (fungal density after antifungal treatment)/(fungal density before antifungal treatment $) \times 100 \%$, as described before $(\mathrm{Wu}$ et al., 2019).

\section{Data Analysis and Statistical Methods}

One-way ANOVA or a non-parametric test was carried out to compare two means, depending on the data distribution. Statistical significance was assumed at a $p$ value of less than 0.05 . Data analysis was performed using Minitab 16 software (Minitab, State College PA, United States).

\section{RESULTS}

\section{C. albicans Forms Epithelium-Associated Biofilms in the Mouse Vagina Accompanied by Fungal Infiltration of the Epithelial Layer}

We examined $C$. albicans laboratory strains and clinical isolates for in vivo biofilm formation on the mouse vaginal epithelium at $72 \mathrm{~h}$ post-inoculation. This time point was chosen because our preliminary experiment using C. albicans DAY185 suggested that fungal invasion and mouse immune response were both evident at this stage of the infection. Qualitative SEM showed that both DAY185 and DAY286 were able to irreversibly attach to the mouse vaginal epithelium, forming monolayer biofilms, with both yeast cells and hyphal elements distributed on the epithelium and apparently internalized by epithelial cells (Figure 1A). Two transcription factor mutant strains that are defective in biofilm formation in vitro, med $31 \Delta \Delta$ and $b \operatorname{cr} 1 \Delta \Delta$, also formed monolayer biofilms on the vaginal epithelium, but to a lower extent (Figure 1A). Parallel quantitative viable counts also suggested biofilm formation of $C$. albicans on the vaginal epithelium, with $\operatorname{med} 31 \Delta \Delta$ and $\operatorname{bcr} 1 \Delta \Delta$ showing densities approximately $1 \mathrm{log} \mathrm{CFU} / \mathrm{g}$ of infected tissue less than that of the wild-type strains (Figure 1B). Periodic acidSchiff staining clearly showed Candida hyphal infiltration of the vaginal epithelial layers. This phenotype, however, was only found for wild-type DAY185 and DAY286, not the mutant strains (Figure 2). Two clinical isolates from patients with vaginal candidiasis also formed significant biofilms on the mouse vaginal epithelium and presented epithelial infiltration, as suggested by SEM imaging, viable counts, and histopathological examination (Figures 1A,B, 2). We also assessed biofilm formation of C. albicans clinical isolates on IUDs, using an in vitro model of biofilm cultivation. At a vaginal physiological $\mathrm{pH}$ of 4.0, C. albicans clinical isolates VVC2 and VVC4 formed threedimensional multilayer biofilms on the surface of IUD wire and in the gaps between neighboring wires (Figure 3 ). The intricate three-dimensional structure of the IUDs, particularly the interwire space, appeared to support robust biofilm formation of C. albicans (Figure 3).

\section{Histopathological Changes of the Vaginal Epithelium Responsive to the Formation of Biotic C. albicans Biofilms and Fungal Infiltration}

One of the key research questions, and also the focus of this study was whether the presence of epithelium-associated biofilms is directly related to the pathological changes within vaginal tissues. We assessed the histopathological changes of the vaginal epithelium and associated alarmin/cytokine responses. Hematoxylin-Eosin staining showed evident neutrophil infiltration and subcorneal microabscesses, two changes of vaginal mucosae typically related to fungal infections in the epithelial layer (Figure 4A). Little change was found when mutant strains $(\operatorname{med} 31 \Delta \Delta$ and $\operatorname{bcr} 1 \Delta \Delta)$ were used to infect 


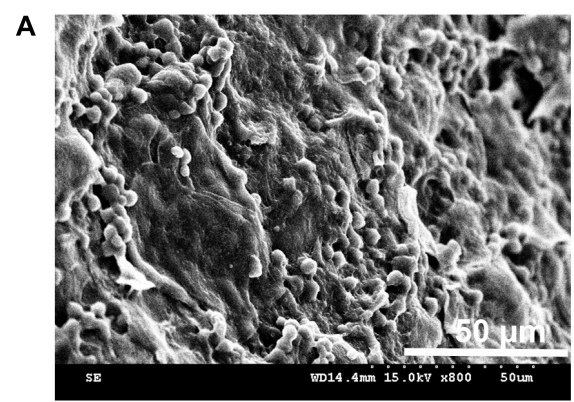

C. albicans DAY185

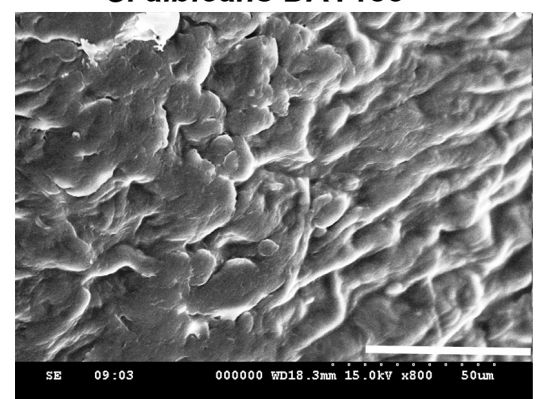

C. albicans med $31 \Delta \Delta$

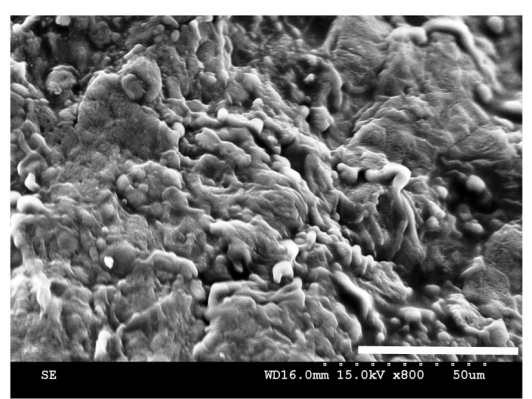

C. albicans DAY286

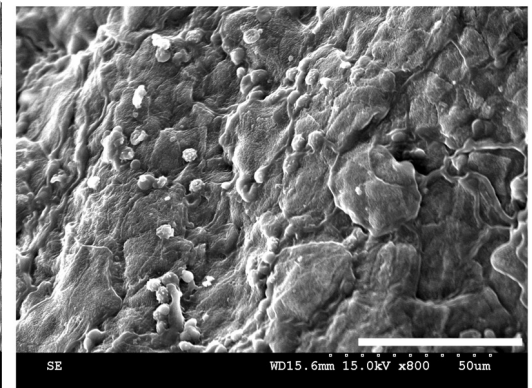

C. albicans bcr1 $1 \Delta \Delta$

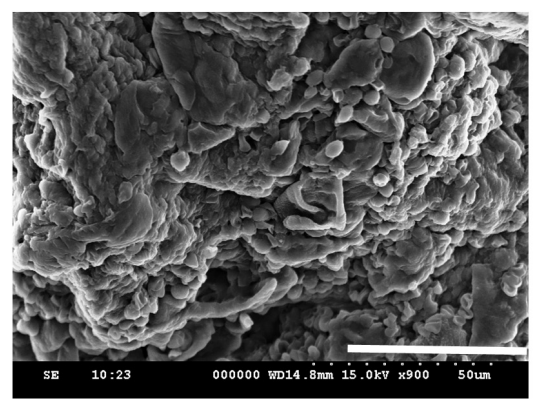

C. albicans VVC2

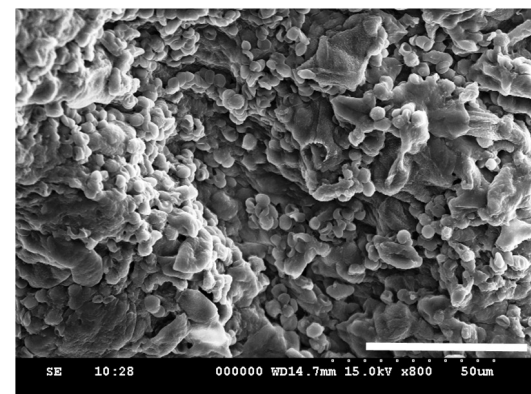

C. albicans VVC4

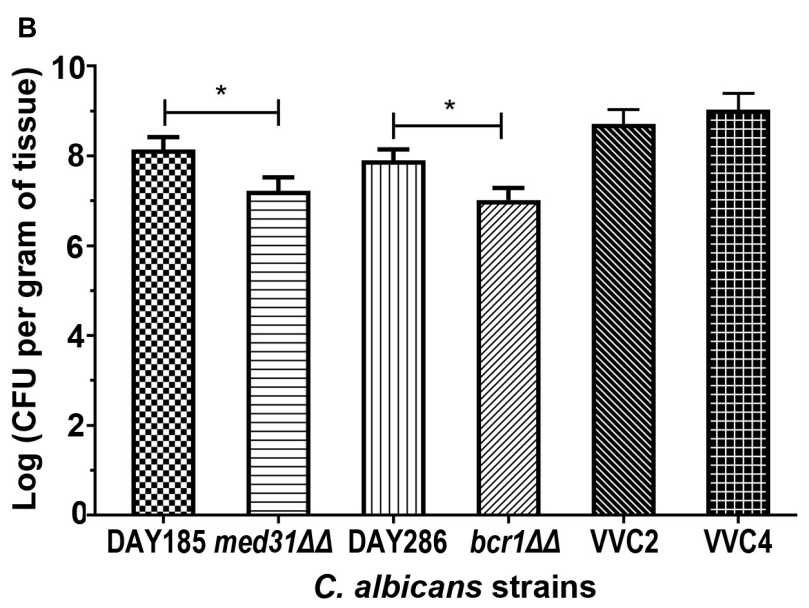

FIGURE 1 | Candida albicans forms biotic biofilms on mouse vaginal mucosae. (A) Scanning electron microscopy of epithelium-associated biotic biofilms formed by C. albicans DAY185 and its med31 $\Delta \Delta$ mutant strain, C. albicans DAY286 and its bcr $1 \Delta \Delta$ mutant strain, and two clinical isolates from patients with vaginal candidiasis. The experiments were repeated on three different occasions. Scale bar $=50 \mu \mathrm{m}$. (B) Quantitative analysis of vaginal epithelium-associated biofilms formed by C. albicans DAY185 and its med31 $\Delta \Delta$ mutant strain, C. albicans DAY286 and its bcr $1 \Delta \Delta$ mutant strain, and two clinical isolates from patients with vaginal candidiasis. CFU-based viable counts were performed. The experiments were repeated three times in triplicate. Means and standard errors were presented. One-way ANOVA was used for two-set comparisons. ${ }^{*} p<0.05$.

the mice (Figure 4A). Enzyme-linked immunosorbent assay targeting cytoplasmic protein S100A8 and cytokine IL-1 $\beta$ demonstrated a raised level of both agents in mice infected with any of the $C$. albicans strains and indicated an inflammatory condition (Figure 4B). C. albicans DAY185 and DAY286 had readings of S100A8 and IL-1 $\beta$ significantly higher than the mutant strains (med $31 \Delta \Delta$ and $b \operatorname{cr} 1 \Delta \Delta$ ), suggesting a possible linkage between the formation of biotic biofilms by $C$. albicans, fungal infiltration, and the harmful immune responses to infected vaginal tissues. Significant histopathological changes and induction of inflammatory responses were also observed when two clinical isolates were introduced to the mouse vagina (Figure 4B).

\section{Formation of Epithelium-Associated Biofilms by $C$. albicans in the Vagina} Leads to Higher Antifungal Resistance

We further tested antifungal susceptibilities of DAY185 and two clinical isolates grown in different modes, including planktonic 

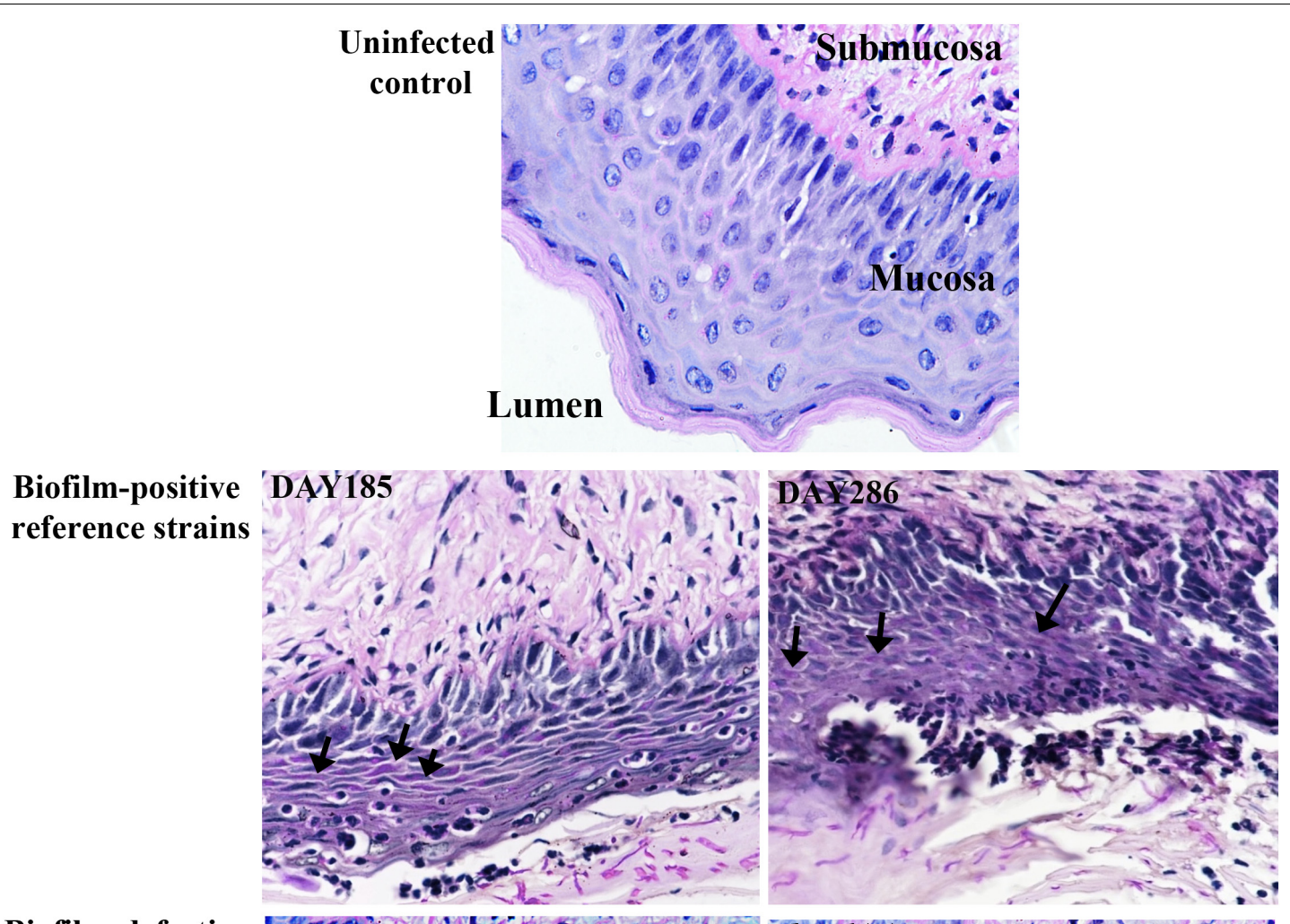

\section{Biofilm-defective mutant strains}
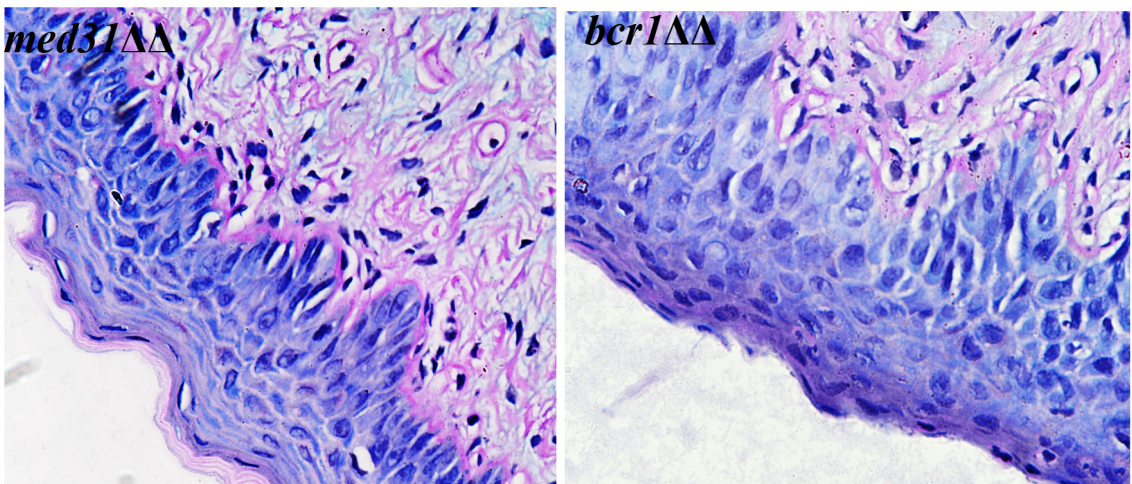

\section{Biofilm-positive clinical isolates}
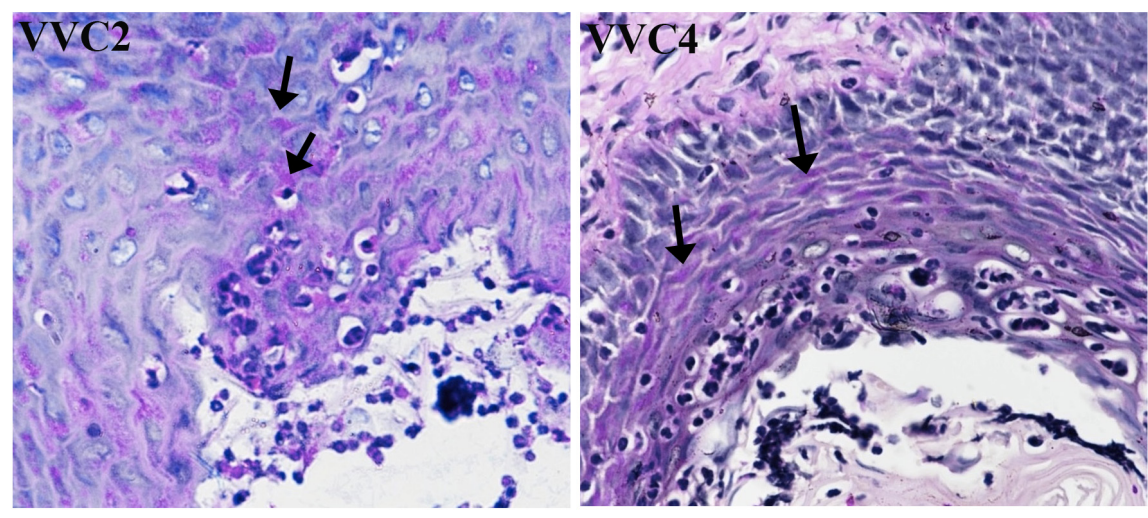

FIGURE 2 | Histology of the vagina of mice infected by C. albicans shows fungal infiltration within the vaginal epithelium. Vaginal histopathological examination (PAS staining, $400 \times$ ) showed that $C$. albicans penetrated the cornified epithelium and formed fungal infiltrations in the mucosal layer. The lumen, mucosa, and submucosa in the uninfected control are denoted for orientation. Evident endocytosed hyphae or fungal infiltrations (black arrows) in the mucosa were observed when C. albicans biofilm-positive reference strains (DAY185 and DAY286) and clinical isolates (WC2 and WC4), but not biofilm-negative mutants (med31 $\Delta \Delta$ and $\operatorname{bcr} 1 \Delta \Delta)$, were used to infect mice. The experiments were repeated on three different occasions. 


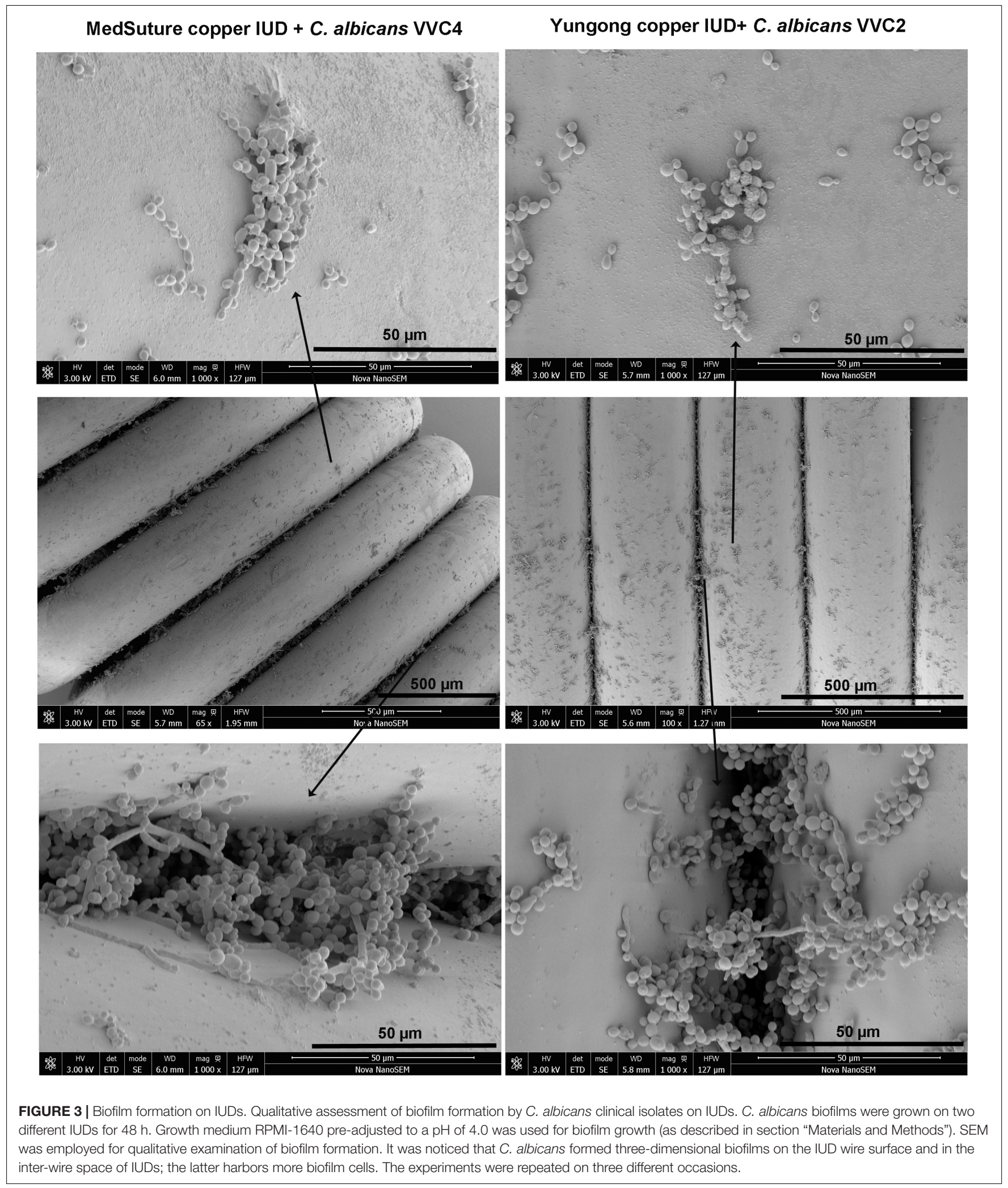

cells, microplate-based abiotic biofilms, and epitheliumassociated biotic biofilms, to three conventional agents under neutral and acidic conditions, respectively (Table 1). At $\mathrm{pH}$
7.2, planktonic cultures of DAY185 and both clinical isolates remained sensitive to all three agents used in this study. Acidic condition of the vagina significantly impacted on the 

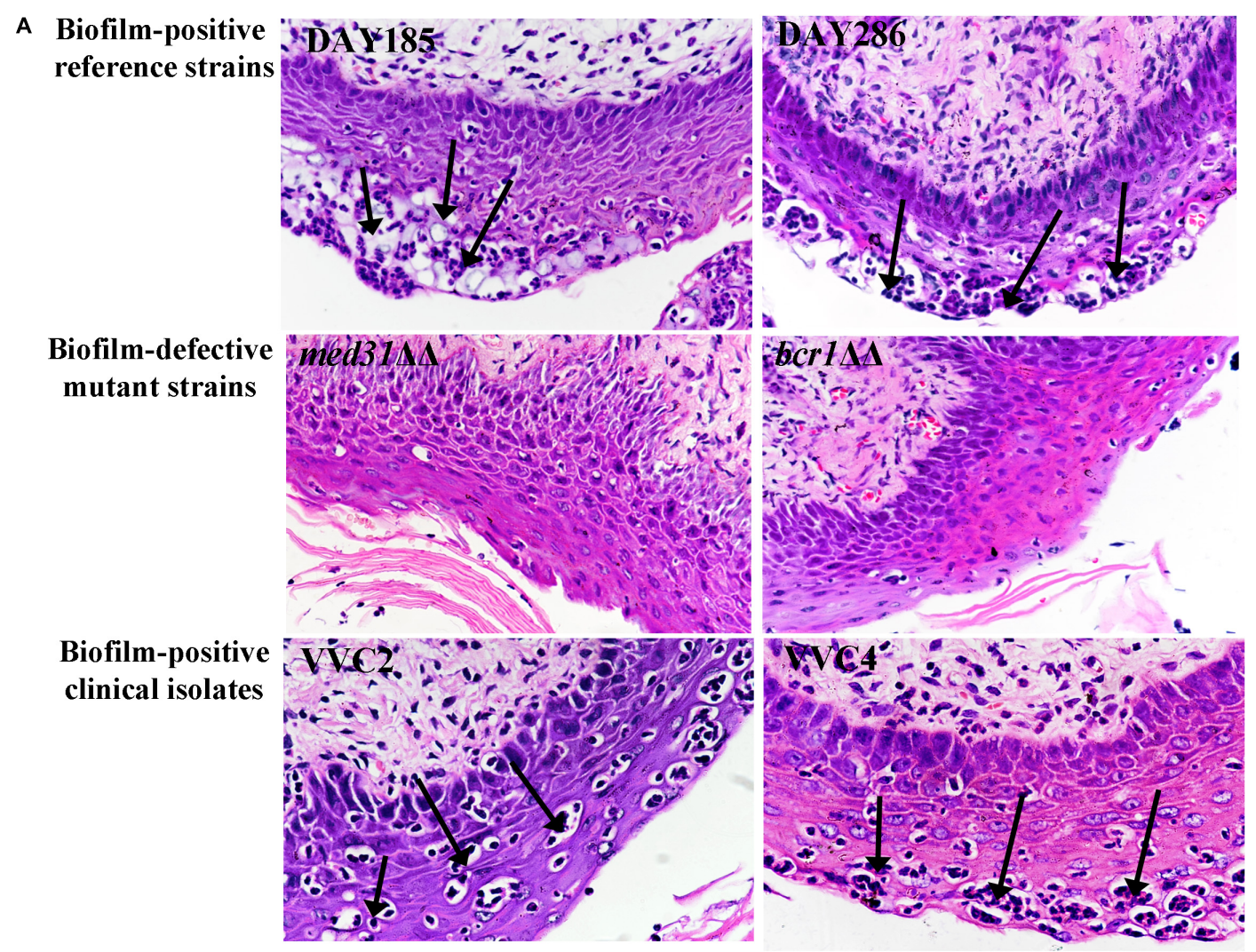

B
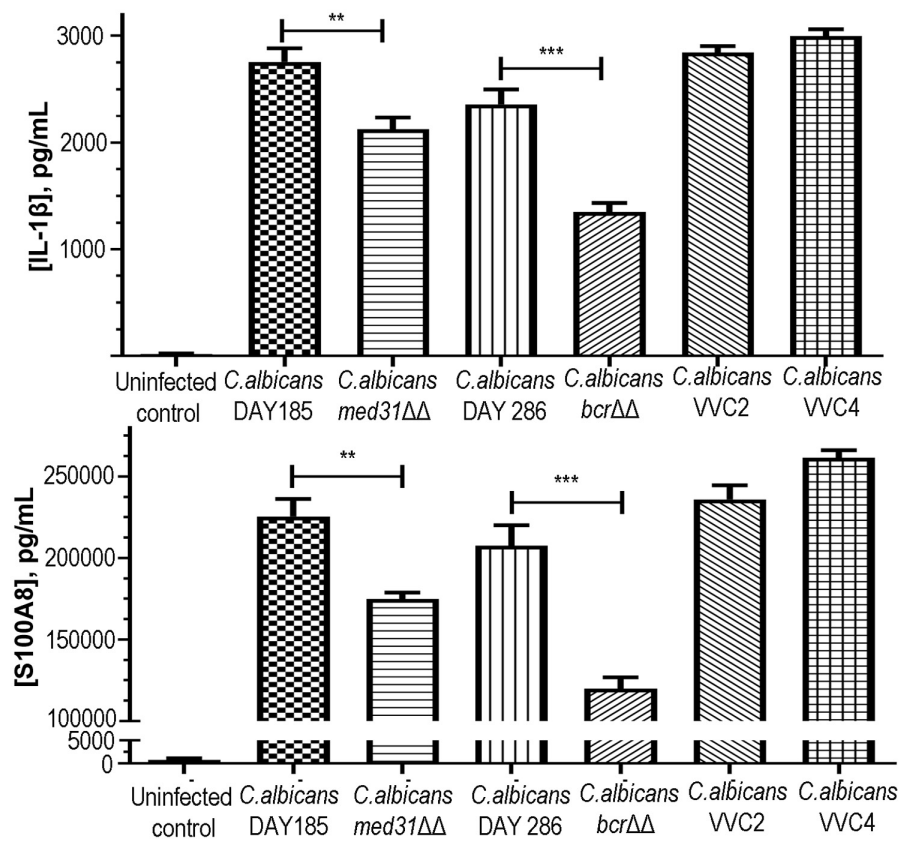

FIGURE 4 | Histopathological and inflammatory changes of the vaginal epithelium after exposure to C. albicans of different biofilm phenotypes. (A) Vaginal histopathological examination (H\&E staining, 400x) showed the formation of numerous microabscesses and neutrophil infiltration (black arrows) in the cornified epithelium after mice were infected with biofilm-positive C. albicans reference strains (DAY185 and DAY286) and clinical isolates (VVC2 and WC4). Minimal neutrophil infiltration and microabscesses were seen when biofilm-defective mutant strains (med31 $\Delta \Delta$ and bcr1 $\Delta \Delta$ ) were used to infect the mice. The experiments were repeated on three different occasions. (B) Local inflammatory responses to C. albicans infections in the mouse vagina. C. albicans DAY185 and its med31 $\Delta \Delta$ mutant strain, C. albicans DAY286 and its bcr1 $\Delta \Delta$ mutant strain, and two clinical isolates from patients with vaginal candidiasis were tested respectively. Cytoplasmic protein S100A8 and cytokine IL-1 $\beta$ were selected as the representative inflammatory effectors. The experiments were repeated three times in duplicate. Means and standard errors were shown. One-way ANOVA or a non-parametric test was used for two-set comparisons. ${ }^{* \star} p<0.01,{ }^{* \star *} p<0.001$. 
TABLE 1 | Antifungal susceptibility of C. albicans: planktonic cells, microplate-based abiotic biofilms, and vaginal epithelium-associated biotic biofilms.

\begin{tabular}{|c|c|c|c|c|c|c|c|c|c|}
\hline \multirow[t]{3}{*}{ Antifungals } & \multicolumn{3}{|c|}{ Planktonic MIC (mg/l) } & \multicolumn{3}{|c|}{ Abiotic biofilm MIC80 (mg/l) } & \multicolumn{3}{|c|}{ Biotic biofilm MIC80 (mg/l) } \\
\hline & \multicolumn{3}{|c|}{ C. albicans strains } & \multicolumn{3}{|c|}{ C. albicans strains } & \multicolumn{3}{|c|}{ C. albicans strains } \\
\hline & DAY185 & Vvc2 & Vvc4 & DAY185 & Vvc2 & Vvc4 & DAY185 & Vvc2 & Vvc4 \\
\hline \multicolumn{10}{|l|}{ pH 7.2} \\
\hline Nystatin & 4 & 2 & 4 & 32 & 64 & 64 & 32 & $>32$ & $>32$ \\
\hline Clotrimazole & 0.5 & 2 & 1 & $>1280$ & $>1280$ & $>1280$ & 1280 & 1280 & $>1280$ \\
\hline Amphotericin B & 1 & 2 & 1 & 1 & 4 & 4 & 2 & 16 & 16 \\
\hline \multicolumn{10}{|l|}{ pH 4.0} \\
\hline Nystatin & 16 & 32 & 32 & 16 & 64 & 128 & 32 & 32 & $>32$ \\
\hline Clotrimazole & 8 & 8 & 16 & 1280 & $>1280$ & $>1280$ & $>1280$ & $>1280$ & $>1280$ \\
\hline Amphotericin B & 2 & 2 & 4 & 2 & 4 & 16 & 2 & $>16$ & $>16$ \\
\hline
\end{tabular}

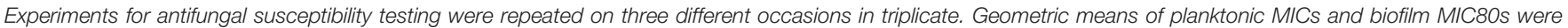
calculated and presented in the table. Definitions and detailed methods for planktonic MIC and biofilm MIC80 can be found in the main text.

susceptibility of nystatin and clotrimazole but not amphotericin B, increasing MICs of nystatin and clotrimazole by 4-16 times. When grown as abiotic biofilms, C. albicans strains generally gained higher resistance to clotrimazole (ratio biofilm MIC80/planktonic MIC: > 160-fold) and nystatin (1- to 16-fold) and remained relatively sensitive to amphotericin B (1- to 4-fold). Epithelium-associated biotic biofilms demonstrated highly elevated resistance to antifungal agents, with two clinical isolates showing biofilm MIC80 even higher than that of microplate-based abiotic biofilms (Table 1).

\section{Formation of Epithelium-Associated Biotic Biofilms by C. albicans Prompts the Formation of Persister Cells}

Persister cells residing in planktonic cultures, microplate-based abiotic biofilms, and epithelium-associated biotic biofilms were quantified. When grown as planktonic cultures at a mid-log phase, C. albicans DAY185 and two clinical isolates produced only very few persister cells (0.0001-0.0008\%; Figure 5). Formation of abiotic biofilms by $C$. albicans on biomaterials significantly increased the number of persister cells in the population; the percentage seemed to be strain-dependent with DAY185 reaching $0.05 \%$, VVC2 reaching $0.1 \%$, and VVC4 reaching $1 \%$. Growth of biotic biofilms on the vaginal epithelium further promoted persister cells of VVC2 to $0.6 \%$, and VVC4 to $3.3 \%$, significantly higher than that for abiotic biofilms or planktonic cultures (Figure 5).

\section{DISCUSSION}

Biofilm formation has been proposed as one of the most important virulence factors of $C$. albicans causing vaginal candidiasis, contributing to the establishment and recurrence of the infection (Muzny and Schwebke, 2015). Although biofilm formation of $C$. albicans has been extensively studied with clinical isolates from vaginal candidiasis patients, most studies used in vitro systems for biofilm cultivation that might only represent biofilms formed on IUDs (Cakiroglu et al., 2015;
Gao et al., 2016; Sherry et al., 2017). Only a few studies have been carried out on biofilms grown on the vaginal epithelium (Harriott et al., 2010; Peters et al., 2014). Harriott et al. (2010) characterized in vivo biofilm formation of C. albicans on the vaginal epithelium; Peters et al. (2014) discovered a link between fungal morphogenesis of $C$. albicans and the immunopathology of vaginal candidiasis (Peters et al., 2014). Using the mouse model developed by Harriott et al. (2010), we further dissected the role of $C$. albicans biofilms in the histopathogenesis and persistence of vaginal candidiasis. Key findings of our study include the following: C. albicans infects the vagina by establishing epithelium-associated biofilms and fungal infiltration; histopathological changes of the vaginal epithelium and local inflammation are in relation to the formation of epithelium-associated biofilms; biofilm formation by C. albicans on the vaginal epithelium leads to high antifungal resistance; formation of biotic biofilms on the vaginal epithelium promotes the formation of persister cells.

By comparing biofilm-positive C. albicans reference strains and their biofilm-defective mutant strains, we established a link between biofilm formation and the disease state of vaginal candidiasis. C. albicans wild-type reference strain DAY286 and its bcr $1 \Delta \Delta$ mutant have been previously studied for in vivo biofilm formation on the mouse vaginal epithelium (Nobile et al., 2006; Harriott et al., 2010). Bcr1 is a transcription factor regulating many important biological processes in C. albicans; its mutant demonstrates normal filamentation but a reduction in biofilm formation on abiotic substrate and host organs (Nobile et al., 2006). Another wild-type reference strain C. albicans DAY185 experimentally causes systematic infection in mice; its med31 deletion mutant showed a reduction in both filamentation and biofilm formation (Uwamahoro et al., 2012). Our study found that biofilm-positive wild-type reference strains, not the biofilmdefective mutants, induced evident histopathological damages and local inflammation, supporting an important role of biofilm formation in the histopathogenesis of vaginal candidiasis. One hurdle in attributing biofilm formation of C. albicans to its pathogenesis is to exclude other co-existing virulence strategies that might also be involved in C. albicans pathogenicity (Sudbery, 


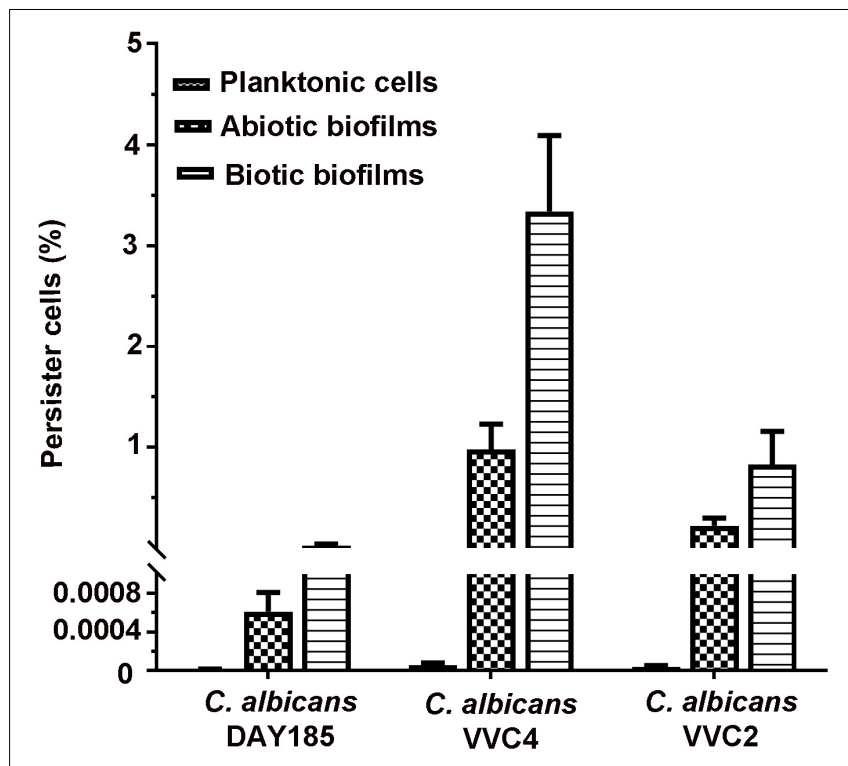

FIGURE 5 | Quantitative assessment of persister cells formed by C. albicans in three different growth modes. C. albicans cells were grown as planktonic cells, abiotic biofilms on tissue culture-treated polystyrene surfaces, and biotic biofilms on the mouse vaginal epithelium, respectively. Biofilm cells and planktonic cells were re-suspended to a density of $\sim 3 \times 10^{7} \mathrm{CFU} / \mathrm{ml}$ in RPMI-1640, followed by exposure to amphotericin B at $100 \mathrm{mg} / \mathrm{l}$ overnight. Survivors were recovered after $72 \mathrm{~h}$ incubation on YPD plates and counted as the percentage of the initial population. The experiments were repeated three times in triplicate. Means and standard errors were presented.

2011). Most transcription factors or their encoding genes often synchronously regulate biofilm formation of $C$. albicans and other important programs such as yeast-hyphae morphogenesis (Nobile et al., 2006; Sudbery, 2011; Uwamahoro et al., 2012). The pathogenesis of vaginal candidiasis has been essentially linked to the hyphal invasive form of $C$. albicans that is required to penetrate the intact epithelial layer and induce immunopathology (Sudbery, 2011). Fortunately, the $b \operatorname{cr} 1 \Delta \Delta$ mutant, which has a defect in biofilm formation but not morphogenesis (Peters et al., 2014), demonstrated a reduced capacity to elicit an immune response or histopathological damage, as found in this study. We also noticed a relation between biofilm formation and candida infiltration of the vaginal epithelium, with biofilmdefective mutant strains presenting less fungal infiltration. Recent work by Swidsinski et al. (2019) found endocytosed hyphae or pseudohyphae in the vaginal epithelium in patients with confirmed vaginal candidiasis, although no typical multi-layer biofilm structure was detected in the human vagina (Swidsinski et al., 2019). We propose that the biofilm-related fungal infiltration might resemble microcolony biofilms that are more frequently found in vivo, and share some important traits with in vivo biofilms, such as harboring antifungal-tolerant persister cells (Bjarnsholt et al., 2013; Xu et al., 2019). It is now known that dense microbial growth in a confined space promotes the formation of persister cells (Qu et al., 2010).

We also presented experimental evidence to explain the tolerance of vaginal candidiasis to antifungal treatment. Similar to microplate-based abiotic biofilms, epithelium-associated biotic biofilms are highly resistant to first-line antifungals. More importantly, epithelium-associated biofilms act as a conducive environment that facilitates the formation of persister cells. Using a regimen that kills the bulk of fungal cells with normal susceptibilities, our recent study has successfully isolated persister cells from the vaginal epithelium of mice infected with C. albicans (Wu et al., 2019). These persister cells could not be eradicated by conventional antifungal drugs at very high concentrations and might be the main culprit responsible for the recalcitrance of infections to antifungal treatment (Wu et al., 2019). The current study further established a connection between biofilm growth by $C$. albicans in the vaginal epithelium and the formation of persister cells, supporting a role of Candida biofilm formation in the recurrence of vaginal candidiasis.

One of the possible limitations of this study was that the mouse model of vaginal candidiasis still differs from human vaginal candidiasis in several physical aspects, including a lack of $C$. albicans as part of the vaginal microbiota, neutral vaginal $\mathrm{pH}$, and dependence on exogenous estrogen to initiate fungal colonization (Harriott et al., 2010; Cassone and Sobel, 2016). Differences in responses to Candida infection between human and mice or other rodents cannot be neglected (Sobel, 2015; Vecchiarelli et al., 2015; Cassone and Sobel, 2016). This small animal model, however, remains a valuable research tool to study vaginal candidiasis as it closely parallels the chronic nature of the diseases in women and is cost-effective (Vecchiarelli et al., 2015; Yano et al., 2018). Another limitation is the small number of clinical isolates used in this study. The conclusion drawn from this study might not fully represent vaginal candidiasis caused by other C. albicans clinical isolates or other Candida spp. The cost, labor, and requirements from the ethics aspect have limited the use of a greater number of C. albicans strains in our study.

\section{CONCLUSION}

In summary, our study provides a comprehensive understanding of biofilm-related factors involved in the pathogenicity and persistence of vaginal candidiasis caused by C. albicans.

\section{DATA AVAILABILITY STATEMENT}

The datasets generated for this study are available on request to the corresponding author.

\section{ETHICS STATEMENT}

The animal study was reviewed and approved by Ethics Committee of Wenzhou Medical University, China.

\section{AUTHOR CONTRIBUTIONS}

YQ and XW conceived and designed the study. SZ, HL, CD, LS, YS, HC, BX, WZ, and YQ carried out the experiments. YQ, SZ, 
$\mathrm{XW}$, and MD performed data analysis. YQ wrote the manuscript. YQ, MD, and XW edited the manuscript. All authors reviewed the manuscript and provided critical comments.

\section{FUNDING}

This work was supported by the National Natural Science Foundation of China (grant number 81772241 to YQ), the National Natural Science Foundation of China (grant number

\section{REFERENCES}

Adjapong, G., Hale, M., and Garrill, A. (2017). A comparative investigation of azole susceptibility in Candida isolates from vulvovaginal candidiasis and recurrent vulvovaginal candidiasis patients in Ghana. Med. Mycol. 55, 686-689.

Auler, M. E., Morreira, D., Rodrigues, F. F., Abr Ao, M. S., Margarido, P. F., Matsumoto, F. E., et al. (2010). Biofilm formation on intrauterine devices in patients with recurrent vulvovaginal candidiasis. Med. Mycol. 48, 211-216. doi: $10.3109 / 13693780902856626$

Bjarnsholt, T., Alhede, M., Alhede, M., Eickhardt-Sorensen, S. R., Moser, C., Kuhl, M., et al. (2013). The in vivo biofilm. Trends Microbiol 21, 466-474.

Blostein, F., Levin-Sparenberg, E., Wagner, J., and Foxman, B. (2017). Recurrent vulvovaginal candidiasis. Ann. Epidemiol. 27, 575.e-582.e.

Bruno, V. M., Shetty, A. C., Yano, J., Fidel, P. L. Jr., Noverr, M. C., et al. (2015). Transcriptomic analysis of vulvovaginal candidiasis identifies a role for the NLRP3 inflammasome. mBio 6:e0182-15.

Cakiroglu, Y., Caliskan, S., Doger, E., Ozcan, S., and Caliskan, E. (2015). Does removal of CU-IUD in patients with biofilm forming candida really maintain regression of clinical symptoms? J. Obstet. Gynaecol. 35, 600-603. doi: 10.3109/ 01443615.2014 .986442

Cassone, A., and Sobel, J. D. (2016). Experimental models of vaginal candidiasis and their relevance to human candidiasis. Infect. Immun. 84, 1255-1261. doi: 10.1128/iai.01544-15

Gamarra, S., Morano, S., Dudiuk, C., Mancilla, E., Nardin, M. E., De Los Angeles Mendez, E., et al. (2014). Epidemiology and antifungal susceptibilities of yeasts causing vulvovaginitis in a teaching hospital. Mycopathologia 178, 251-258. doi: $10.1007 /$ s11046-014-9780-2

Gao, M., Wang, H., and Zhu, L. (2016). Quercetin assists fluconazole to inhibit biofilm formations of fluconazole-resistant Candida albicans in in vitro and in vivo antifungal managements of vulvovaginal candidiasis. Cell Physiol. Biochem. 40, 727-742. doi: 10.1159/000453134

Gargani, G., Zecchi Orlandi, S., Campisi, E., Pini, G., and Orlandini, G. E. (1989). Scanning electron microscopic pattern of recurrent vaginitis by Candida albicans in the mouse. Mycoses 32, 644-651. doi: 10.1111/j.1439-0507.1989. tb02197.x

Goncalves, B., Ferreira, C., Alves, C. T., Henriques, M., Azeredo, J., and Silva, S. (2016). Vulvovaginal candidiasis: epidemiology, microbiology and risk factors. Crit. Rev. Microbiol. 42, 905-927. doi: 10.3109/1040841x.2015.1091805

Hagerty, J. A., Ortiz, J., Reich, D., and Manzarbeitia, C. (2003). Fungal infections in solid organ transplant patients. Surg. Infect. 4, 263-271. doi: 10.1089/ 109629603322419607

Harriott, M. M., Lilly, E. A., Rodriguez, T. E., Fidel, P. L. Jr., and Noverr, M. C. (2010). Candida albicans forms biofilms on the vaginal mucosa. Microbiology 156, 3635-3644. doi: 10.1099/mic.0.039354-0

Ilkit, M., and Guzel, A. B. (2011). The epidemiology, pathogenesis, and diagnosis of vulvovaginal candidosis: a mycological perspective. Crit. Rev. Microbiol 37, 250-261. doi: 10.3109/1040841x.2011.576332

Kalkanci, A., Guzel, A. B., Jabban, I., Aydin, M., Ilkit, M., and Kustimur, S. (2013). Candida vaginitis in non-pregnant patients: a study of antifungal susceptibility testing and virulence factors. J. Obstet. Gynaecol. 33, 378-383. doi: 10.3109/ 01443615.2013.767323

Kasper, L., Miramon, P., Jablonowski, N., Wisgott, S., Wilson, D., Brunke, S., et al. (2015). Antifungal activity of clotrimazole against Candida albicans depends on
81873822 to XW), and the Shenzhen Science and Technology Innovation Projects (grant numbers JCY20170818100355169 and JCY201908083000479 to XW).

\section{ACKNOWLEDGMENTS}

The authors wish to acknowledge Prof. Ana Traven from Monash University for kindly donating $C$. albicans strains including DAY286, DAY185, med31 $\Delta \Delta$, and $b \operatorname{cr} 1 \Delta \Delta$.

carbon sources, growth phase and morphology. J. Med. Microbiol. 64, 714-723. doi: $10.1099 /$ jmm.0.000082

Kojic, E. M., and Darouiche, R. O. (2004). Candida infections of medical devices. Clin. Microbiol. Rev. 17, 255-267. doi: 10.1128/cmr.17.2.255-267.2004

Lafleur, M. D., Kumamoto, C. A., and Lewis, K. (2006). Candida albicans biofilms produce antifungal-tolerant persister cells. Antimicrob. Agents Chemother. 50, 3839-3846. doi: 10.1128/aac.00684-06

LaFleur, M. D., Qi, Q., and Lewis, K. (2010). Patients with long-term oral carriage harbor high-persister mutants of Candida albicans. Antimicrob. Agents Chemother. 54, 39-44. doi: 10.1128/aac.00860-09

Lewis, K. (2010). Persister cells. Annu. Rev. Microbiol. 64, 357-372.

Liu, X. P., Fan, S. R., Peng, Y. T., and Zhang, H. P. (2014). Species distribution and susceptibility of Candida isolates from patient with vulvovaginal candidiasis in Southern China from 2003 to 2012. J. Mycol. Med. 24, 106-111. doi: 10.1016/j. mycmed.2014.01.060

Muzny, C. A., and Schwebke, J. R. (2015). Biofilms: an underappreciated mechanism of treatment failure and recurrence in vaginal infections. Clin. Infect. Dis. 61, 601-606. doi: 10.1093/cid/civ353

Nagashima, M., Yamagishi, Y., and Mikamo, H. (2016). Antifungal susceptibilities of Candida species isolated from the patients with vaginal candidiasis. J. Infect. Chemother. 22, 124-126. doi: 10.1016/j.jiac.2015.08.008

Nobile, C. J., Andes, D. R., Nett, J. E., Smith, F. J., Yue, F., Phan, Q. T., et al. (2006). Critical role of Bcrl-dependent adhesins in C. albicans biofilm formation in vitro and in vivo. PLoS Pathog. 2:e63. doi: 10.1371/journal.ppat.002 0063

Peters, B. M., Palmer, G. E., Nash, A. K., Lilly, E. A., Fidel, P. L. Jr., et al. (2014). Fungal morphogenetic pathways are required for the hallmark inflammatory response during Candida albicans vaginitis. Infect. Immun. 82, 532-543. doi: 10.1128/iai.01417-13

Qu, Y., Daley, A. J., Istivan, T. S., Rouch, D. A., and Deighton, M. A. (2010). Densely adherent growth mode, rather than extracellular polymer substance matrix build-up ability, contributes to high resistance of Staphylococcus epidermidis biofilms to antibiotics. J. Antimicrob. Chemother. 65, 1405-1411. doi: 10.1093/ jac/dkq119

Qu, Y., Locock, K., Verma-Gaur, J., Hay, I. D., Meagher, L., and Traven, A. (2016). Searching for new strategies against polymicrobial biofilm infections: guanylated polymethacrylates kill mixed fungal/bacterial biofilms. J. Antimicrob. Chemother. 71, 413-421. doi: 10.1093/jac/dkv334

Richardson, J. P., Willems, H. M. E., Moyes, D. L., Shoaie, S., Barker, K. S., Tan, S. L., et al. (2017). Candidalysin drives epithelial signaling, neutrophil recruitment, and immunopathology at the vaginal mucosa. Infect. Immun. 86, e645-e617.

Richter, S. S., Galask, R. P., Messer, S. A., Hollis, R. J., Diekema, D. J., and Pfaller, M. A. (2005). Antifungal susceptibilities of Candida species causing vulvovaginitis and epidemiology of recurrent cases. J. Clin. Microbiol. 43, 2155-2162. doi: 10.1128/jcm.43.5.2155-2162.2005

Rodriguez-Cerdeira, C., Gregorio, M. C., Molares-Vila, A., Lopez-Barcenas, A., Fabbrocini, G., Bardhi, B., et al. (2019). Biofilms and vulvovaginal candidiasis. Colloids Surf. B Biointerfaces 174, 110-125.

Sherry, L., Kean, R., Mckloud, E., O’donnell, L. E., Metcalfe, R., Jones, B. L., et al. (2017). Biofilms formed by isolates from recurrent vulvovaginal candidiasis patients are heterogeneous and insensitive to fluconazole. Antimicrob. Agents Chemother. 61:e01065-17. 
Sobel, J. D. (2015). Editorial commentary: Vaginal biofilm: much ado about nothing, or a new therapeutic challenge? Clin. Infect. Dis. 61, 607-608. doi: $10.1093 / \mathrm{cid} / \mathrm{civ} 358$

Sobel, J. D. (2016). Recurrent vulvovaginal candidiasis. Am. J. Obstet. Gynecol. 214, $15-21$.

Sudbery, P. E. (2011). Growth of Candida albicans hyphae. Nat. Rev. Microbiol. 9, 737-748. doi: 10.1038/nrmicro2636

Swidsinski, A., Guschin, A., Tang, Q., Dorffel, Y., Verstraelen, H., Tertychnyy, A., et al. (2019). Vulvovaginal candidiasis: histologic lesions are primarily polymicrobial and invasive and do not contain biofilms. Am. J. Obstet. Gynecol. 220:e91.

Uwamahoro, N., Qu, Y., Jelicic, B., Lo, T. L., Beaurepaire, C., Bantun, F., et al. (2012). The functions of Mediator in Candida albicans support a role in shaping species-specific gene expression. PLoS Genet. 8:e1002613. doi: 10.1371/journal. pgen. 1002613

Vecchiarelli, A., Gabrielli, E., and Pericolini, E. (2015). Experimental models of vaginal candidiasis and inflammation. Future Microbiol. 10, 1265-1268. doi: $10.2217 / \mathrm{fmb} .15 .52$

Wu, X., Zhang, S., Xu, X., Shen, L., Xu, B., Qu, W., et al. (2019). RAFTderived polymethacrylates as a superior treatment for recurrent vulvovaginal candidiasis by targeting biotic biofilms and persister cells. Front. Microbiol. 10:2592. doi: 10.3389/fmicb.2019.02592

Xu, B., Deighton, M., and Qu, Y. (2019). Should we absolutely reject the hypothesis that epithelium-based Candida biofilms contribute to the pathogenesis of human vulvovaginal candidiasis?. Am. J. Obst. Gynecol. 221, 372-373. doi: 10.1016/j.ajog.2019.07.007

Yang, S., Hay, I. D., Cameron, D. R., Speir, M., Cui, B., Su, F., et al. (2015). Antibiotic regimen based on population analysis of residing persister cells eradicates Staphylococcus epidermidis biofilms. Sci. Rep. 5:18578.

Yano, J., Peters, B. M., Noverr, M. C., and Fidel, P. L. Jr. (2018). Novel mechanism behind the immunopathogenesis of vulvovaginal candidiasis: "neutrophil anergy". Infect. Immun. 86:e00684-17.

Ying, C., Zhang, H., Tang, Z., Chen, H., Gao, J., and Yue, C. (2016). Antifungal susceptibility and molecular typing of 115 Candida albicans isolates obtained from vulvovaginal candidiasis patients in 3 Shanghai maternity hospitals. Med. Mycol. 54, 394-399. doi: 10.1093/mmy/myv082

Conflict of Interest: The authors declare that the research was conducted in the absence of any commercial or financial relationships that could be construed as a potential conflict of interest.

Copyright (C) $2020 \mathrm{Wu}$, Zhang, Li, Shen, Dong, Sun, Chen, Xu, Zhuang, Deighton and $\mathrm{Qu}$. This is an open-access article distributed under the terms of the Creative Commons Attribution License (CC BY). The use, distribution or reproduction in other forums is permitted, provided the original author(s) and the copyright owner(s) are credited and that the original publication in this journal is cited, in accordance with accepted academic practice. No use, distribution or reproduction is permitted which does not comply with these terms. 